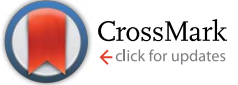

Cite this: RSC Adv., 2017, 7, 6080
Received 26th October 2016 Accepted 7th January 2017

DOI: $10.1039 / c 6 r a 25843 c$

\section{Polymyxin B sulfate inducing time-dependent antagonism of the mixtures of pesticide, ionic liquids, and antibiotics to Vibrio qinghaiensis sp.-Q67†}

\begin{abstract}
Ye Fan, ${ }^{a}$ Shu-Shen Liu, ${ }^{\star a b}$ Rui Qu, ${ }^{a}$ Kai Li ${ }^{\mathrm{a}}$ and Hai-Ling Liu*b
In the real environment, organisms are simultaneously exposed to different types of chemicals. Different types of chemicals usually have different time-dependent toxicity characteristics, however, people know little about the time-dependent toxicities of mixtures. Hence, the time-dependent toxicity of one pesticide, two ionic liquids (ILs), two antibiotics 20 ternary mixture rays and ten quaternary mixture rays to Vibrio qinghaiensis sp.-Q67 (V. qinghaiensis) were determined by the time-dependent microplate toxicity analysis. The results showed that the toxicity of pesticide (imidacloprid, IMI) to $V$. qinghaiensis does not change with time, the toxicities of two ILs (1-hexyl-3-methyl-imidazolium bromide, [hmim] Br, and 1-hexyl-3-methylimidazolium chloride, [hmim] Cl) decrease with time, and those of two antibiotics (chloramphenicol, $\mathrm{CHL}$, and polymyxin B sulfate, POL) increase with time. The mixture toxicity almost doesn't change with time. Taking the concentration addition model as an additive reference, the combined toxicities of chemicals are assessed. It is shown that the mixture toxicities of most rays in two ternary mixture systems, [hmim] $\mathrm{Br}-\mathrm{IMI}-\mathrm{CHL}$ and $[\mathrm{hmim}] \mathrm{Cl}-\mathrm{IMI}-\mathrm{CHL}$, are additive at any time, while those in $[\mathrm{hmim}] \mathrm{Br}-\mathrm{IMI}-\mathrm{POL}$ and $[\mathrm{hmim}] \mathrm{Cl}-\mathrm{IMI}-\mathrm{POL}$ systems are antagonistic at some time, which implies that the POL may be a key component inducing the antagonism. To further validate the antagonism induced by $\mathrm{POL}$, the $\mathrm{POL}$ is respectively merged into two ternary $[\mathrm{hmim}] \mathrm{Br}-\mathrm{IMI}-\mathrm{CHL}$ and $[\mathrm{hmim}] \mathrm{Cl}-\mathrm{IMI}-\mathrm{CHL}$ systems having no antagonism to form two quaternary mixture systems. It is found that many rays in the two new quaternary mixture systems have antagonistic action at some times, which validates that POL is indeed a key component. These findings indicate that the mixture toxicity of pesticide, ILs and antibiotics is complicated and prone to generate interaction, and doing risk assessment should take this type of mixtures into consideration.
\end{abstract}

\section{Introduction}

The effects of toxicants on test organisms are determined by chemical dose, and are also related to exposure duration. If only the short-term bioassay is taken into consideration, it is possible to underestimate or overrate the toxicity of some substances. ${ }^{1-3}$ For example, Backhaus et al. took Vibrio fischeri as test organisms to determine the short-term toxicities (30 $\mathrm{min}$ ) and the long-term toxicities $(24 \mathrm{~h})$ of antibiotics and found that the toxicity of the antibiotic increased significantly with time. ${ }^{4}$ Zhu et al. determined the short-term and the long-term toxicities of six triazine herbicides on Vibrio qinghaiensis sp.-Q67 ( $V$. qinghaiensis), and showed that the short-term toxicities of

${ }^{a}$ Key Laboratory of Yangtze River Water Environment, Ministry of Education, College of Environmental Science and Engineering, Tongji University, Shanghai 200092, China. E-mail: ssliuhl@263.net

${ }^{b}$ State Key Laboratory of Pollution Control and Resource Reuse, College of Environmental Science and Engineering, Tongji University, Shanghai 200092, China $\dagger$ Electronic supplementary information (ESI) available. See DOI: 10.1039/c6ra25843c desmetryne and simetryn are similar to their long-term toxicities, and the long-term toxicities of velpar, prometon and metribuzin are higher than their short-term toxicities, and aminotriazine has significant long-term toxicity but no shortterm toxicity. ${ }^{5}$ Liu et al. examined the short-term and longterm toxicities of two pesticides (imidacloprid and pirimicarb) and antibiotic (streptomycin sulfate) on $V$. qinghaiensis, and indicated that the short-term toxicities of pesticides are similar to their long-term toxicities, and the long-term toxicity of antibiotic is higher than its short-term toxicity. ${ }^{6}$ Some people also found that the short-term toxicities of ionic liquids (1-ethyl-3methylimidazolium chloride and 1-butyl-3-methylimidazolium chloride) are higher than their long-term toxicities to $V$. qinghaiensis. ${ }^{7}$ All of these indicate that different types of chemicals have different characteristics of the time-dependent toxicity.

Regulatory risk assessment of chemicals usually focuses on single chemicals, while chemicals usually co-occur in real environment. ${ }^{8}$ In recent years, there were many reports on mixture toxicity. ${ }^{6,9-11}$ Pulicharla et $a l .{ }^{12}$ selected chlortetracycline (CTC) and some metals as the mixture 
components to investigate the mixture toxicity of CTC and metal and found that the mixtures are more toxic than the CTC. Aronzon et al. examined the joint toxicity of binary mixtures of $\mathrm{Cu}$ and diazion on the South American toad Rhinella arenarum and showed that the mixture toxicity is antagonistic at almost all conditions. ${ }^{13}$ Geiger et $a .^{14}$ researched the mixture toxicity of pharmaceuticals and chlorophenols to freshwater algae Chlorella vulgaris and concluded that the mixture toxicities are higher than those of each mixture component. Also, some researchers explored the combined toxicities of ternary and multiplecomponent mixtures. ${ }^{15,16}$ However, further research is needed on mixture toxicity of the chemicals having different timedependent toxicity profiles.

It has been shown that some pesticides, antibiotics and ionic liquids (ILs) have different time-dependent toxicity profiles. Pesticide pollution has become a global issue. ${ }^{17,18}$ Over the past few decades, the negative effects about the use and handling of drugs on human has gradually attracted people's attention. ${ }^{\mathbf{1 9 2 0}}$ An antibiotic as a kind of pharmaceuticals is a chemotherapeutic agent that inhibits or abolishes the growth of microorganisms, such as bacteria, fungi, or protozoa which has been proved to produce toxicology effect on the animals in the natural water bodies. ${ }^{21}$ ILs are organic salts with low melting point that are being considered as green replacements for industrial volatile organic compounds, however, ILs still pose serious threats to aquatic and terrestrial ecosystems. ${ }^{22,23}$ Then, what combined toxicity do the chemicals having different timedependent toxicity profiles produce when they are mixed, which need to be further studied. It is found, the toxicity of pesticide (imidacloprid, IMI) to $V$. qinghaiensis does not change with time, the toxicities of two ILs (1-hexyl-3-methylimidazolium bromide, [hmim] Br, and 1-hexyl-3-methyl-imidazolium chloride, [hmim]Cl) decrease with time, and those of two antibiotics (chloramphenicol, CHL, and polymyxin B sulfate, POL) increase with time. Meanwhile, the five chemicals also have environmental meaning. Camp et al. ${ }^{24}$ indicated that IMI has been detected worldwide at different concentrations. $\mathrm{Xu}$ et $a l^{25}$ detected $\mathrm{CHL}$ in the Pearl River at Guangzhou during high and low water seasons. Ding et $a .^{26}$ found that POL is existing in pharmaceutical wastewaters. Although there are almost no reports on ILs existing in the real environment, the knowledge of ILs is equally important since both soil and aquatic milieu are possible recipients of ILs contamination. ${ }^{22}$ So, the five chemicals having different timedependent toxicity profiles were selected as mixture components to construct many mixture systems. For each mixture system, five rays with different concentration ratios were designed by using the uniform design ray procedure (UDray). ${ }^{27}$ For each mixture ray, 12 mixture points with different<smiles>O=[N+]([O-])/N=C1\NCCN1Cc1ccc(Cl)nc1</smiles>

Imidacloprid<smiles>CCCCCCn1cc[n+](C)c1</smiles>

1-Hexyl-3-methylimidazolium bromide<smiles>O=C(N[C@H](CO)[C@H](O)c1ccc([N+](=O)[O-])cc1)C(Cl)Cl</smiles>

Chloramphenicol<smiles>CCCCCCn1cc[n+](C)c1</smiles>

1-Hexyl-3-methylimidazolium chloride

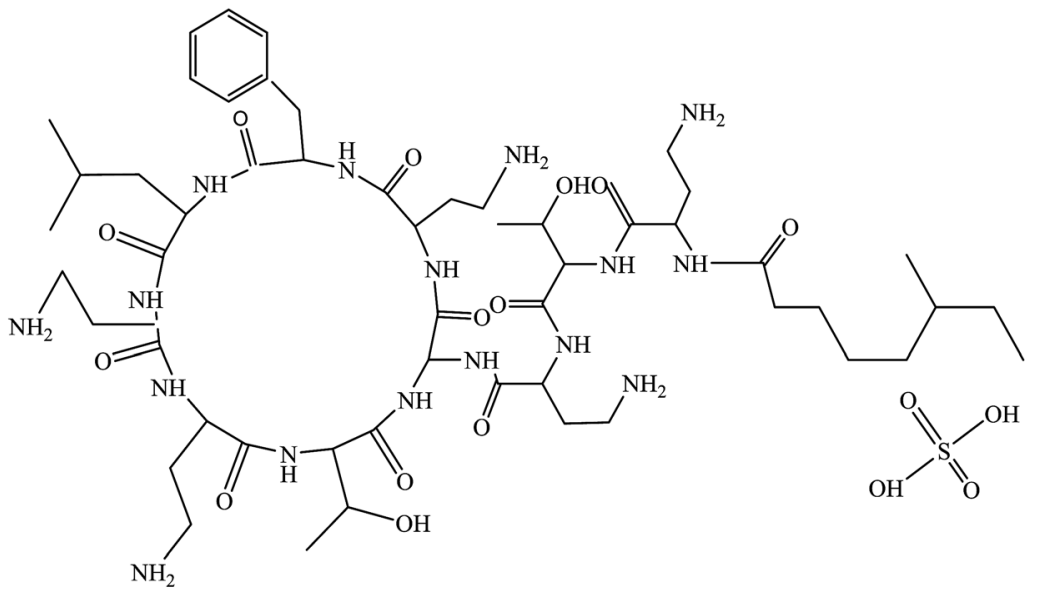

Polymyxin B sulfate

Fig. 1 The molecular structures of $\mathrm{IMI}$, [hmim]Br, [hmim] Cl, CHL, and POL. 
concentration levels were specified by the fixed ratio ray design (FRRD). ${ }^{28}$ The toxicities of single chemicals and various mixture rays to $V$. qinghaiensis at five time points such as 0.25 , $3,6,9$ and $12 \mathrm{~h}$ were determined by the time-dependent microplate toxicity analysis (t-MTA). ${ }^{29}$ Taking the concentration addition (CA) model as an additive reference, the toxicity interactions in mixtures were evaluated.

\section{Materials and methods}

\section{Chemicals}

Two ILs, [hmim] $\mathrm{Br}$ and [hmim]Cl, were purchased from TCI (Japan). One pesticide, IMI, and one antibiotic, CHL, were purchased from Dr Ehrenstorfer (Germany). POL was purchased from TRC (Canada). All solutions were prepared with Milli-Q water and stored in darkness at $4{ }^{\circ} \mathrm{C}$ before test. Some physical properties and the concentrations of stocks were listed in Table S1 of the ESI. $\dagger$ And the molecular structures of the chemicals were shown in Fig. 1.

\section{Time-dependent toxicity test}

In this paper, $V$. qinghaiensis was selected as the test organism. The freeze-dried $V$. qinghaiensis was purchased from Beijing
Hamamatsu Corp., Ltd. (Beijing, China). Medium formula for $V$. qinghaiensis, the culture condition, and relative light unit determination are the same as those in the literature, ${ }^{30}$ and the only difference is that the set of time points is at $0.25,3,6,9$ and $12 \mathrm{~h}$. In the same way, the toxicity of a chemical or mixture ray to $V$. qinghaiensis is expressed as an inhibition ratio of bioluminescence.

\section{Design of mixtures}

Taking one pesticide (IMI), two ILs ([hmim] Br and [hmim]Cl) and two antibiotics (CHL and POL) as mixture components, four ternary mixture systems ([hmim]Br-IMI-CHL, [hmim]Cl-IMICHL, [hmim]Br-IMI-POL, and [hmim]Cl-IMI-POL) were constructed. Because a mixture system includes a lot of mixture rays, ${ }^{30}$ the UD-ray ${ }^{27,31,32}$ was used to select some representative mixture rays to explore systematically the combined toxicity of mixture system. For each mixture system, the five effective concentrations $\left(\mathrm{EC}_{10}, \mathrm{EC}_{20}, \mathrm{EC}_{30}, \mathrm{EC}_{40}\right.$ and $\left.\mathrm{EC}_{50}\right)$ of each component were selected as five levels. According to the uniform table, $\mathrm{U}_{5}\left(5^{4}\right)$ where the subscript (5) refers to the number of the mixture rays, the number 5 refers to the number of level of each component and the superscript (4) refers to the maximum number of components, five rays (noted as R1, R2, R3, R4 and R5)

Table 1 Concentration ratios $(p)$ of various components in various mixture rays and the concentrations of stocks of various mixture rays

Mixture ratios of components $(p)$

IMI

$[\mathrm{hmim}] \mathrm{Br}$

$[\mathrm{hmim}] \mathrm{Cl}$

CHL

POL stock $\left(\mathrm{mol} \mathrm{L}^{-1}\right)$

\begin{tabular}{|c|c|}
\hline No. & Mixture ray \\
\hline \multicolumn{2}{|c|}{ Ternary mixture ray $(T)$} \\
\hline $\mathrm{T} 1$ & [hmim]Br-IMI-CHL-R1 \\
\hline $\mathrm{T} 2$ & [hmim] $\mathrm{Br}-\mathrm{IMI}-\mathrm{CHL}-\mathrm{R} 2$ \\
\hline T3 & [hmim] Br-IMI-CHL-R3 \\
\hline $\mathrm{T} 4$ & [hmim] $\mathrm{Br}-\mathrm{IMI}-\mathrm{CHL}-\mathrm{R} 4$ \\
\hline T5 & [hmim]Br-IMI-CHL-R5 \\
\hline T6 & [hmim]Cl-IMI-CHL-R1 \\
\hline $\mathrm{T} 7$ & [hmim]Cl-IMI-CHL-R2 \\
\hline T8 & [hmim]Cl-IMI-CHL-R3 \\
\hline Т9 & [hmim]Cl-IMI-CHL-R4 \\
\hline $\mathrm{T} 10$ & [hmim]Cl-IMI-CHL-R5 \\
\hline T11 & [hmim]Br-IMI-POL-R1 \\
\hline T12 & [hmim] Br-IMI-POL-R2 \\
\hline $\mathrm{T} 13$ & [hmim]Br-IMI-POL-R3 \\
\hline T14 & [hmim]Br-IMI-POL-R4 \\
\hline T15 & [hmim] Br-IMI-POL-R5 \\
\hline T16 & [hmim]Cl-IMI-POL-R1 \\
\hline T17 & [hmim]Cl-IMI-POL-R2 \\
\hline $\mathrm{T} 18$ & [hmim]Cl-IMI-POL-R3 \\
\hline T19 & [hmim]Cl-IMI-POL-R4 \\
\hline $\mathrm{T} 20$ & [hmim]Cl-IMI-POL-R5 \\
\hline
\end{tabular}

Quaternary mixture ray $(\mathrm{Q})$

Q1 [hmim]Br-IMI-CHL-POL-R1

Q2 [hmim]Br-IMI-CHL-POL-R2

Q3 [hmim]Br-IMI-CHL-POL-R3

Q4 [hmim]Br-IMI-CHL-POL-R4

Q5 [hmim]Br-IMI-CHL-POL-R5

Q6 [hmim]Cl-IMI-CHL-POL-R1

Q7 [hmim]Cl-IMI-CHL-POL-R2

Q8 [hmim]Cl-IMI-CHL-POL-R3

Q9 [hmim]Cl-IMI-CHL-POL-R4

Q10 [hmim]Cl-IMI-CHL-POL-R5

$$
\begin{aligned}
& 1.449 \times 10^{-1} \\
& 2.331 \times 10^{-1} \\
& 3.510 \times 10^{-2} \\
& 1.133 \times 10^{-1} \\
& 1.929 \times 10^{-1} \\
& 1.230 \times 10^{-1} \\
& 2.160 \times 10^{-1} \\
& 3.373 \times 10^{-2} \\
& 1.135 \times 10^{-1} \\
& 1.991 \times 10^{-1} \\
& 1.445 \times 10^{-1} \\
& 2.328 \times 10^{-1} \\
& 3.502 \times 10^{-2} \\
& 1.131 \times 10^{-1} \\
& 1.927 \times 10^{-1} \\
& 1.227 \times 10^{-1} \\
& 2.157 \times 10^{-1} \\
& 3.366 \times 10^{-2} \\
& 1.134 \times 10^{-1} \\
& 1.988 \times 10^{-1}
\end{aligned}
$$

$$
\begin{aligned}
& 8.548 \times 10^{-1} \\
& 7.668 \times 10^{-1} \\
& 9.647 \times 10^{-1} \\
& 8.866 \times 10^{-1} \\
& 8.069 \times 10^{-1} \\
& \\
& \\
& \\
& 8.520 \times 10^{-1} \\
& 7.656 \times 10^{-1} \\
& 9.627 \times 10^{-1} \\
& 8.854 \times 10^{-1} \\
& 8.059 \times 10^{-1}
\end{aligned}
$$

$8.743 \times 10^{-1}$

$7.828 \times 10^{-1}$

$9.641 \times 10^{-1}$

$8.852 \times 10^{-1}$

$7.997 \times 10^{-1}$

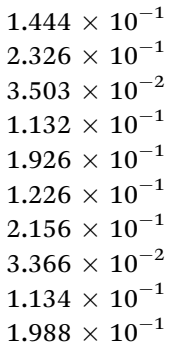

$8.515 \times 10^{-1}$ $7.652 \times 10^{-1}$ $9.629 \times 10^{-1}$ $8.856 \times 10^{-1}$ $8.058 \times 10^{-1}$

$8.739 \times 10^{-1}$ $7.823 \times 10^{-1}$ $9.643 \times 10^{-1}$ $8.853 \times 10^{-1}$ $7.995 \times 10^{-1}$

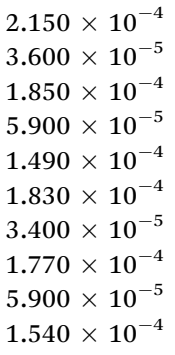

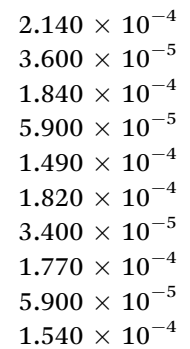

$3.689 \times 10^{-3}$ $4.948 \times 10^{-3}$ $4.174 \times 10^{-3}$ $5.161 \times 10^{-3}$ $4.033 \times 10^{-3}$ $4.707 \times 10^{-3}$ $5.872 \times 10^{-3}$ $4.833 \times 10^{-3}$ $5.756 \times 10^{-3}$ $4.834 \times 10^{-3}$ $2.457 \times 10^{-3}$ $3.098 \times 10^{-3}$ $3.750 \times 10^{-3}$ $4.006 \times 10^{-3}$ $3.421 \times 10^{-3}$ $2.759 \times 10^{-3}$ $3.248 \times 10^{-3}$ $3.806 \times 10^{-3}$ $3.966 \times 10^{-3}$ $3.330 \times 10^{-3}$

$\begin{array}{ll}3.916 \times 10^{-3} & 2.079 \times 10^{-3} \\ 2.165 \times 10^{-3} & 2.719 \times 10^{-3} \\ 1.896 \times 10^{-3} & 3.514 \times 10^{-3} \\ 1.202 \times 10^{-3} & 4.023 \times 10^{-3} \\ 1.465 \times 10^{-3} & 3.040 \times 10^{-3} \\ 3.326 \times 10^{-3} & 2.348 \times 10^{-3} \\ 2.007 \times 10^{-3} & 2.854 \times 10^{-3} \\ 1.822 \times 10^{-3} & 3.569 \times 10^{-3} \\ 1.205 \times 10^{-3} & 3.976 \times 10^{-3} \\ 1.512 \times 10^{-3} & 2.951 \times 10^{-3}\end{array}$


were designed by Table S2 of the ESI $\dagger$ in the literature. ${ }^{27}$ For each mixture ray, 12 mixture points with different concentration levels were specified by FRRD. ${ }^{28}$ The concentration ratios $(p)$ of various components in various mixture rays and the concentrations of stocks of various rays were listed in Table 1. Furthermore, to validate whether the POL is a key component inducing antagonism, two quaternary mixture systems ([hmim]Br-IMI-CHL-POL and [hmim]Cl-IMI-CHL-POL) were constructed.

\section{Concentration-response curve fitting and toxicity interaction analysis}

The Weibull function with the location parameter $(\alpha)$ and shape parameter $(\beta)$ was selected as a fit function for the single substances and mixture rays. ${ }^{31}$ The $95 \%$ observation-based confidence intervals (OCIs) of concentration-response curve
(CRC) fitted were calculated to express the uncertainty in toxicity test and fitting process. ${ }^{33,34}$

The concentration addition (CA) model $^{34}$ was selected as an additive reference to analyze the toxicological interaction in mixtures. When the CRC predicted by the CA is located between the OCIs of experimental CRC, the mixture toxicity is additive. If the predictive CRC is less than the lower limit of the OCI or higher than the upper limit of the OCI, the mixture toxicity is synergistic or antagonistic.

\section{Results and discussion}

\section{Time-dependent toxicity of single chemical}

The CRC features of five single chemicals in five different exposure times was shown in Fig. 2. Obviously, different types of
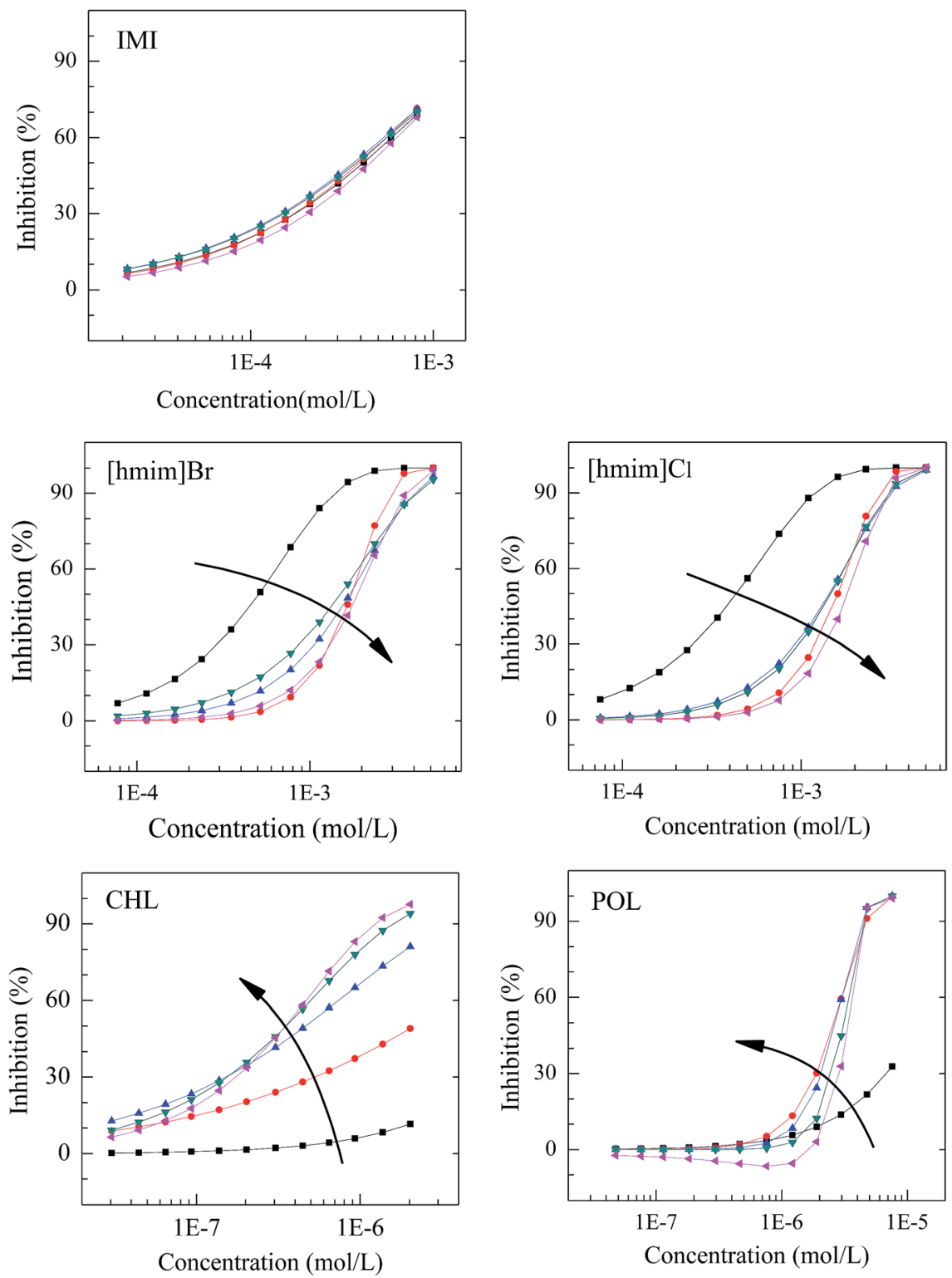

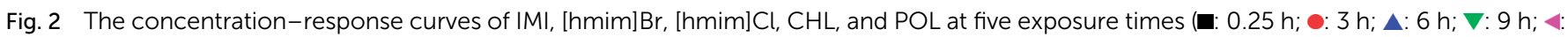
$12 \mathrm{~h} ; \rightarrow$ : toxicity increasing). 

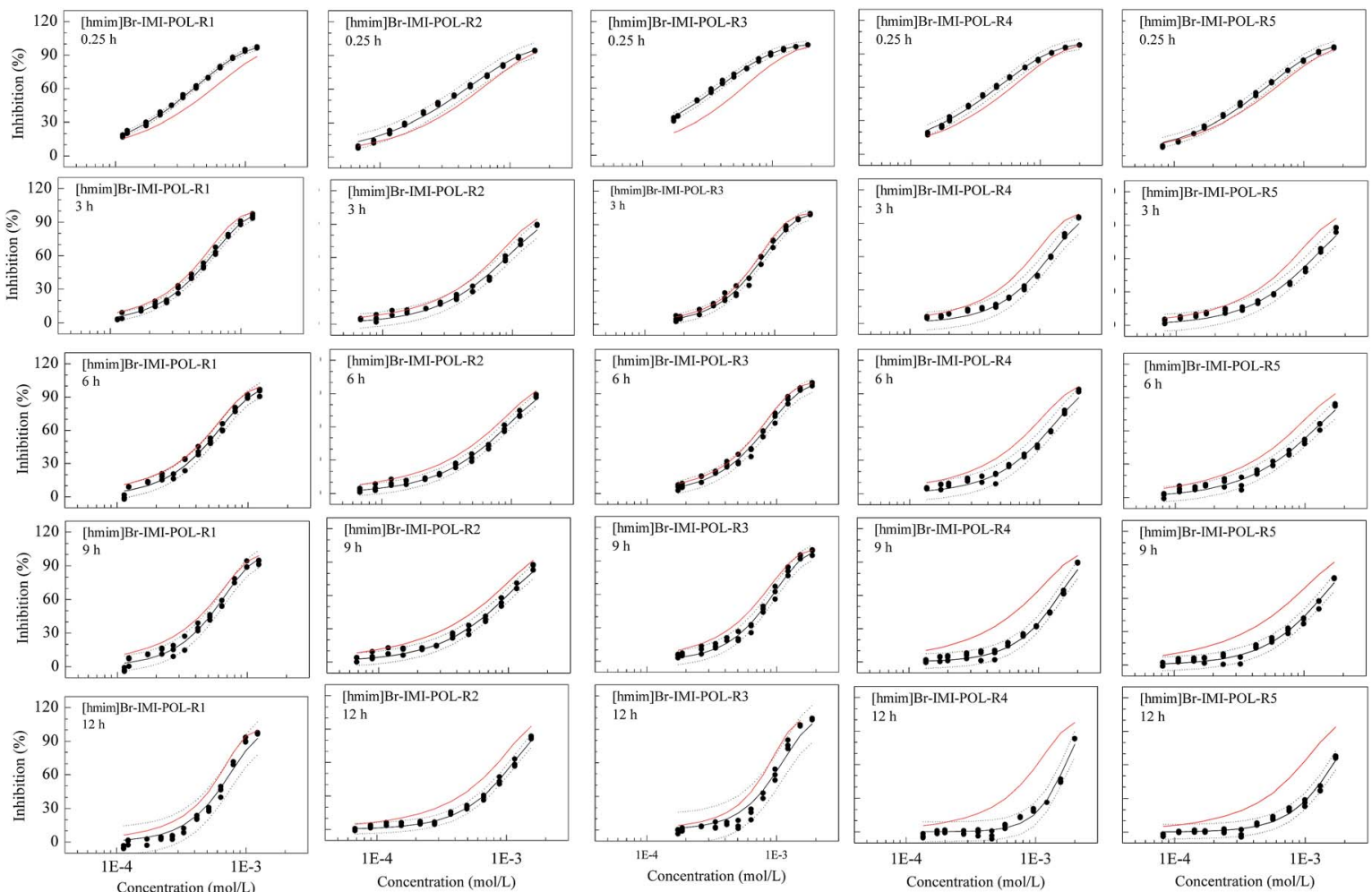

Fig. 3 The concentration-response relationships of five mixture rays (R1, R2, R3, R4 and R5) in [hmim]Br-IMI-POL systems at five exposure times where the black dots $(\mathbf{})$ refer to the experimental value, the black solid lines $(-)$ to those fitted by the Weibull, the red solid lines ( -$)$ to those predicted by CA and the dashed lines (---) to the $95 \%$ confidence intervals.
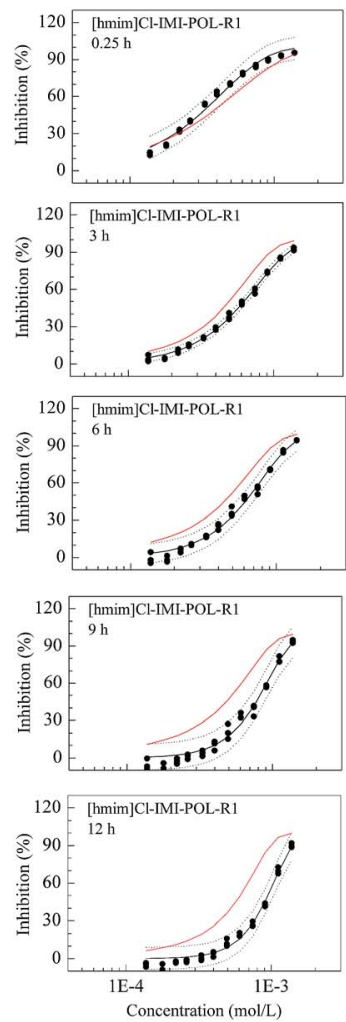
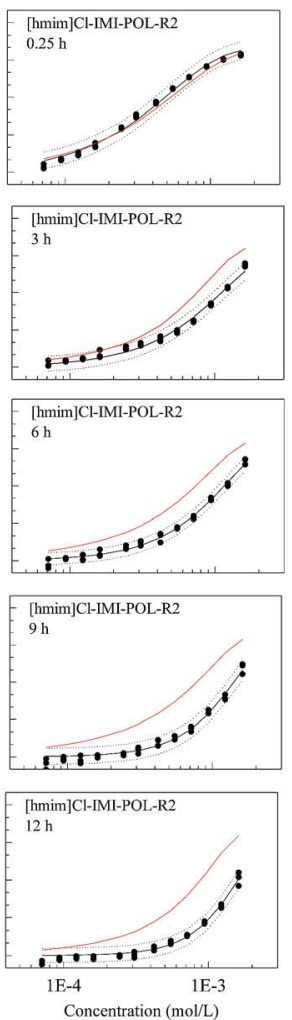
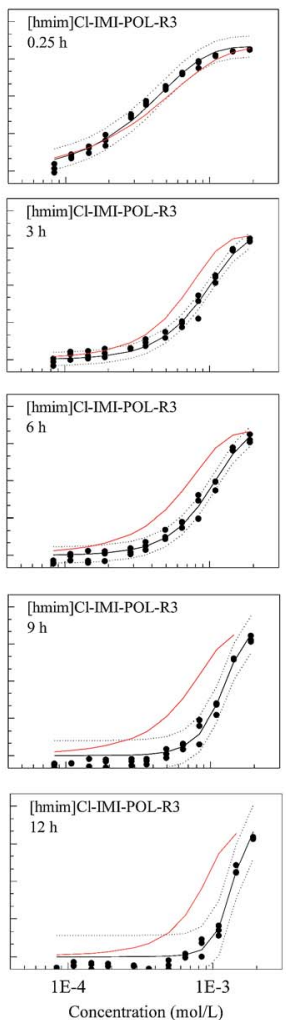
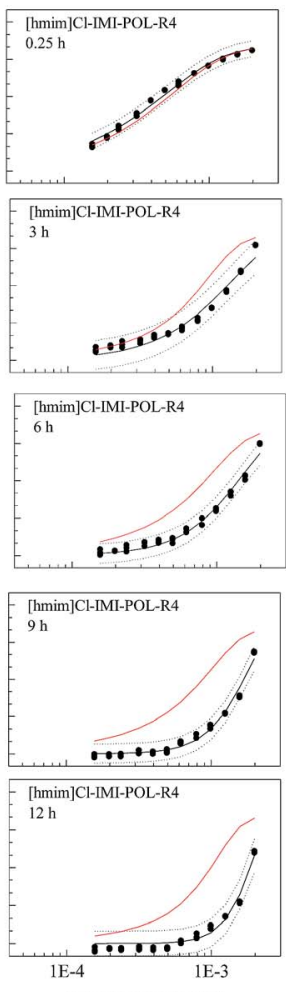

Concentration (mol/L)
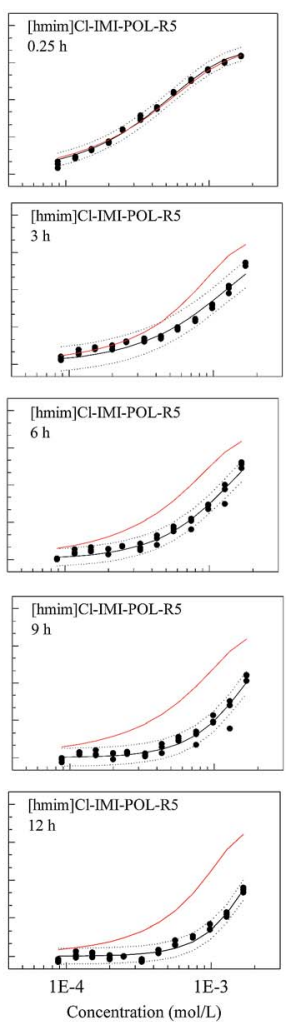

Fig. 4 The concentration-response relationships of five mixture rays (R1, R2, R3, R4 and R5) in [hmim] Cl-IMI-POL systems at five exposure times where the black dots $(\bullet)$ refer to the experimental value, the black solid lines $(-)$ to those fitted by the Weibull, the red solid lines (-) to those predicted by CA and the dashed lines (---) to the $95 \%$ confidence intervals. 
chemicals have different time-dependent toxicity profiles. The CRC of IMI remains unchanged with time, i.e., the toxicity of pesticide doesn't change with time, which is consistent with results reported by Liu et al., ${ }^{6} \mathrm{Qu} e t \mathrm{al}$. also find that the toxicity of pesticide simetryn is unchanged with time. ${ }^{35}$ The CRC of [hmim] $\mathrm{Br}$ and $[\mathrm{hmim}] \mathrm{Cl}$ initially shifts to the right from 0.25 to $3 \mathrm{~h}$ and then the CRCs almost overlap after $3 \mathrm{~h}$, which indicates that the toxicities of ILs decrease initially and then remains unchanged. However, the CRC of CHL and POL initially move to the top-left and then become stable, which shows that the toxicity of antibiotics increases at first and then remains unchanged. The results are similar to those of some antibiotics such as kanamycin sulfate ${ }^{36}$ and streptomycin sulfate. ${ }^{6}$ The fitted CRC model parameter ( $\alpha$ and $\beta)$, statistics ( $R^{2}$ and RMSE) and toxicity index $\left(\mathrm{EC}_{50}\right)$ were listed in Table S2 of the ESI. $\dagger$ From the Table $\mathrm{S} 2, \dagger$ the median effective concentrations $\left(\mathrm{EC}_{50}\right)$ of CHL and POL are 2-3 orders of magnitude lower than those of ILs and pesticide, which manifests that the toxicity of CHL and POL is higher than ILs and pesticide to $V$. qinghaiensis.

\section{Time-dependent toxicity of mixtures}

Although pesticide, ILs and antibiotics have different timedependent toxicity profiles, the toxicity of the ternary mixtures almost does not change with time. Plots of the $\mathrm{pEC}_{20}, \mathrm{pEC}_{50}$ and $\mathrm{pEC}_{70}$ of twenty ternary mixture rays were shown in Fig. S1 of the ESI. $\uparrow$ The toxicities of ten mixture rays in [hmim]Br-IMI-
CHL and [hmim]Cl-IMI-CHL systems is unchanged with time. However, the toxicities of R1 in [hmim] Br-IMI-POL and [hmim] Cl-IMI-POL system is slightly higher than other mixture rays. These results explain that the combined toxicities of chemicals almost have no time-dependance.

The change trend of time-dependent toxicity of the mixture rays in two quaternary mixture systems, [hmim] Br-IMI-CHLPOL and [hmim]Cl-IMI-CHL-POL, is similar to that in the ternary mixtures (Fig. S2 of the ESI†). The toxicity of two quaternary mixtures is almost unchanged. The toxicities of $\mathrm{R} 1$ in [hmim]Br-IMI-CHL-POL and [hmim] Cl-IMI-CHL-POL system is slightly higher than other mixture rays.

However, the toxicities of the mixtures with different concentration ratios (different rays) or different concentration levels (different area on a ray) are different, which illustrate that the combined toxicity have the concentration ratio-dependance and concentration level-dependance..$^{37,38}$

\section{Toxicological interaction in ternary mixtures}

Toxicological interaction in mixtures can be identified by means of the comparison of the experimental toxicity with that predicted by the CA.

For the two ternary mixture systems, [hmim]Br-IMI-CHL and [hmim] Cl-IMI-CHL, the CA prediction toxicity of all mixture rays at various effect levels and all exposure times are located between the OCIs of experimental toxicity (Fig. S3 and
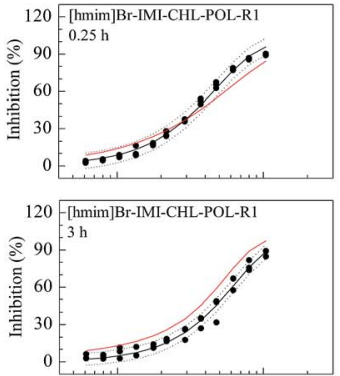

$120-[\mathrm{hmim}] \mathrm{Br}-\mathrm{IMI}-\mathrm{CHL}-\mathrm{POL}-\mathrm{R} 1$
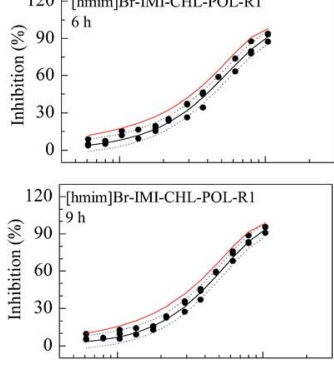

120 [hmim]Br-IMI-CHL-POL-R1

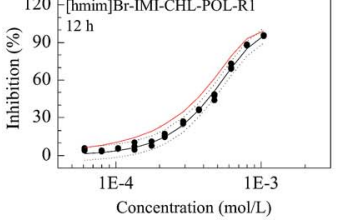

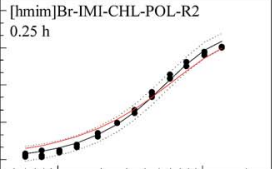

[hmim]Br-IMI-CHL-POL-R2

$3 \mathrm{~h}$
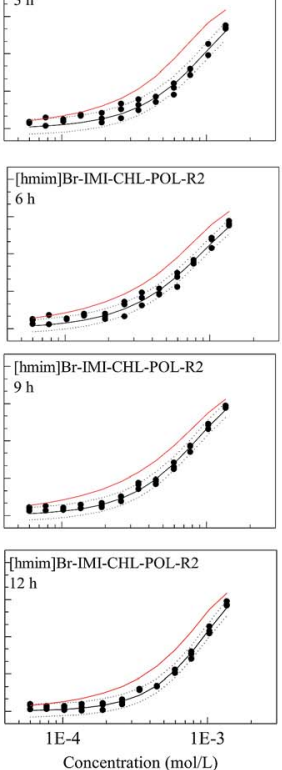
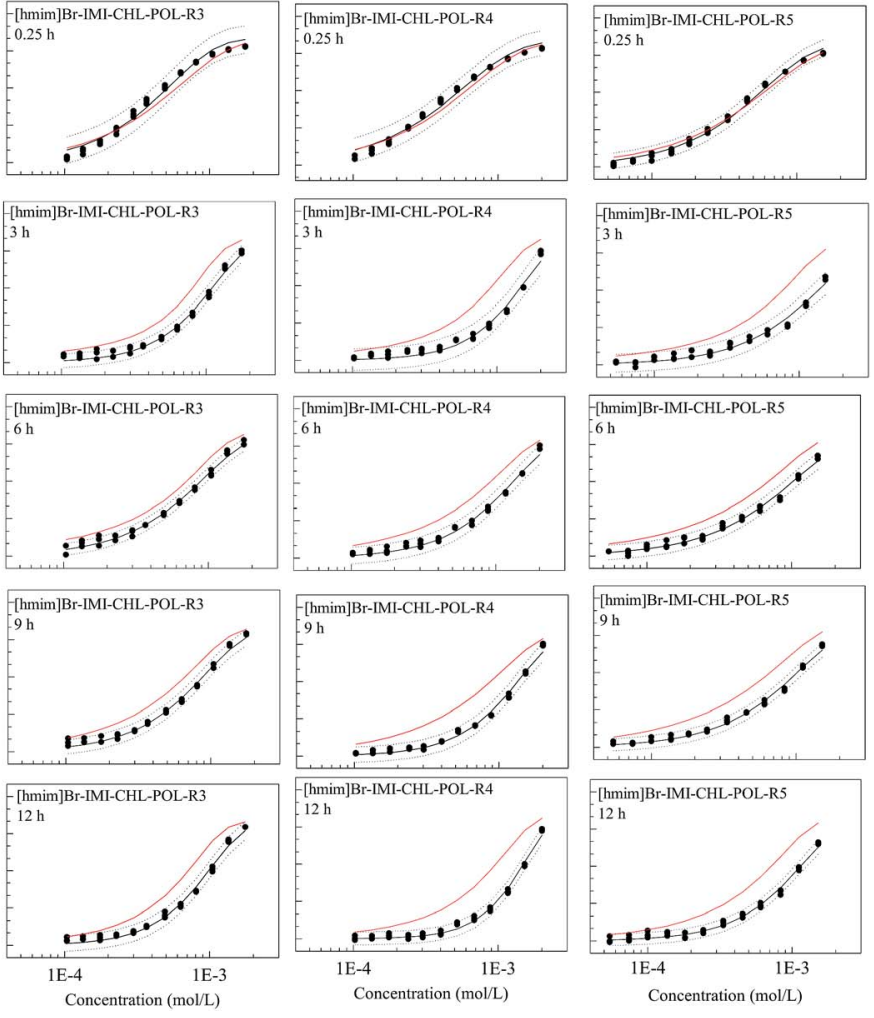

-[hmim]Br-IMI-CHL-POL-R5

$-6 \mathrm{~h}$
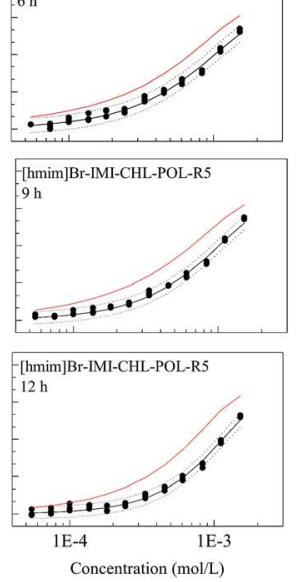

Fig. 5 The concentration-response relationships of five mixture rays (R1, R2, R3, R4 and R5) in [hmim] Br-IMI-CHL-POL systems at five exposure times where the black dots $(\bullet)$ refer to the experimental value, the black solid lines $(-)$ to those fitted by the Weibull, the red solid lines $(-)$ to those predicted by CA and; the dashed lines (---) to the $95 \%$ confidence intervals. 
S4 of the ESI $\dagger$ ), which implies that the toxicities of all mixtures are additive or the mixtures have no synergism or antagonism. That is, the toxicological interactions of all mixtures are additive action, independent of the mixture ratio (different rays), exposure time and concentration level.

However, for the ternary mixture system, [hmim]Br-IMIPOL, some mixture rays such as R4 and R5 at 9 and $12 \mathrm{~h}$ exhibit significant antagonism, while the rays, R1 and R3, at $0.25 \mathrm{~h}$ display synergism, which implies the different rays at different time having different interaction (Fig. 3). That is, the toxicological interactions of mixtures in this ternary system are different and have the mixture ratio-dependance and timedependance. Combined with Table 1 , both the R4 and R5 having less mixture ratios of POL $\left(1.441 \times 10^{-3}\right.$ and $1.465 \times$ $10^{-3}$ ) than the other two rays (R1 and R3) exit significant antagonism at 9 and $12 \mathrm{~h}$. The $\mathrm{R} 2$ with the mixture ratio of 1.615 $\times 10^{-3}$ has also antagonism. In the same time, the ray with less ratio of POL at $0.25 \mathrm{~h}$ presents less synergism or additive action, while the ray with higher ratio of POL at $0.25 \mathrm{~h}$ shows synergism.

This is also true for the ternary [hmim]Cl-IMI-POL mixture system (Fig. 4). In the [hmim]Cl-IMI-POL system, all mixture rays at all times $(3,6,9$ and $12 \mathrm{~h}$ ) except one $(0.25 \mathrm{~h})$ exhibit antagonism and the degree of antagonism increases with time. Unlike the [hmim]Br-IMI-POL system, all mixture rays in the [hmim]Cl-IMI-POL system at $0.25 \mathrm{~h}$ have everywhere additive action.
The reason why the ternary mixtures containing POL show antagonism may be that: in microorganisms, pesticides may interfere with cell growth, division, and molecular composition. ${ }^{39}$ For ILs, toxicity seemed to be determined mainly by the cationic component, Austin et al. explained that the molecules can be intercalated into the membrane, while their ionic head group is at least partially solvated in the aqueous solution. ${ }^{\mathbf{4 0 , 4 1}}$ POL is the narrow-spectrum antibiotic which can inhibit the cell membrane synthesis of Gram-negative bacteria, while CHL belongs to amide alcohol broad spectrum antibiotics which can also inhibit Gram-negative bacteria. ${ }^{26}$ Although $V$. qinghaiensis are Gram-negative, they are sensitive to POL and CHL, the reason why the mixtures which contain POL show antagonism may be that, there is a competition on the cell target site among pesticide-ILs-POL, while the inhibition mechanism of chloramphenicol involves the suppression of transpeptidase activity and the disturbance to the function of 50S ribosome avoiding the competition for the target site.

In general, toxicological interaction in mixtures is complicated. The toxicological interactions of some mixtures are additive action. While combined toxicity of some mixtures are dependent on the mixture ratio and exposure time, and the interaction type (additive action, synergism or antagonism) are different at different mixture ratios and exposure times. The majority of approaches which aim to assess the risk of environmental chemical mixture, including regulatory bodies and the prediction of the mixture toxicity using toxicity data stems
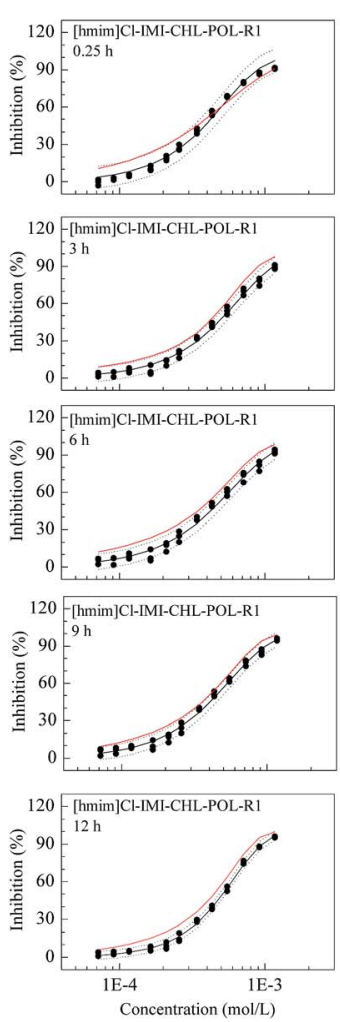

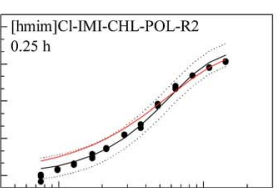

-[hmim]Cl-IMI-CHL-POL-R2 $3 \mathrm{~h}$
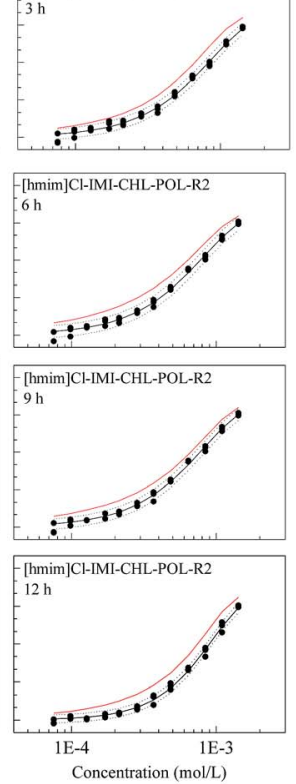
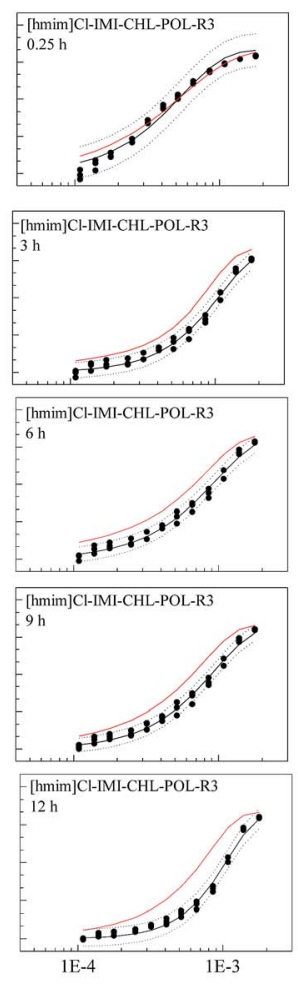

Concentration $(\mathrm{mol} / \mathrm{L})$
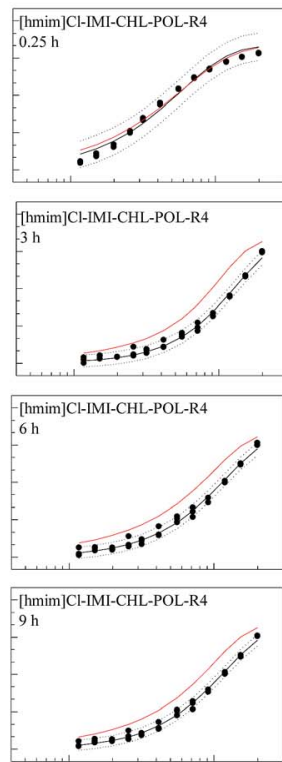

-[hmim]Cl-IMI-CHL-POL-R4

$12 \mathrm{~h}$

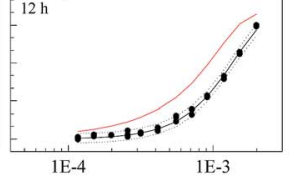

Concentration $(\mathrm{mol} / \mathrm{L})$
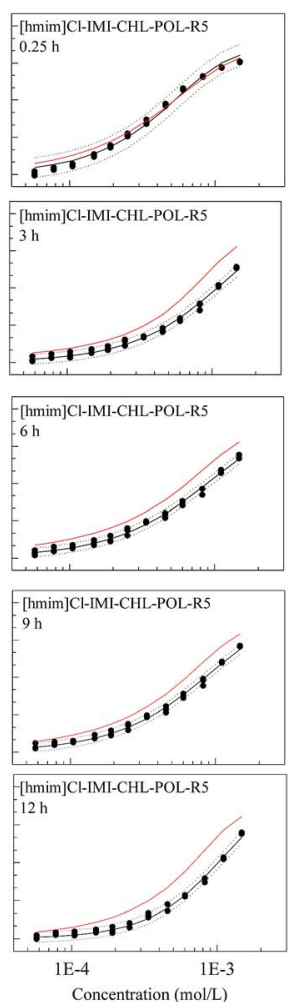

Fig. 6 The concentration-response relationships of five mixture rays (R1, R2, R3, R4 and R5) in [hmim] Cl-IMI-CHL-POL systems at five exposure times where the black dots $(\bullet)$ refer to the experimental value, the black solid lines $(-)$ to those fitted by the Weibull, the red solid lines $(-)$ to those predicted by CA and; the dashed lines (---) to the $95 \%$ confidence intervals. 
from the individual component substance. ${ }^{42}$ Therefore, when the test organism is determined, doing risk assessment for these mixture should take mixture ratios and time into consideration simultaneously.

\section{POL being the key component producing antagonism}

From the toxicological interaction of ternary mixture systems, the change of POL seems to lead to change of interaction, in other words, the POL may be a key component of antagonism. In order to verify the guess, the POL were respectively merged into two ternary mixture systems which have no interactions to form two new quaternary mixture systems, [hmim]Br-IMICHL-POL and [hmim]Cl-IMI-CHL-POL. The results show that most rays produce antagonism with the increasing of time (Fig. 5 and 6), which verifies that POL is indeed a key component which causes time-dependent antagonism of the mixture to $V$. qinghaiensis sp.-Q67.

\section{Conclusions}

We determined time-dependent toxicity of five chemicals, one pesticide (IMI), two ILs ([hmim] Br and [hmim] Cl), and two antibiotics (CHL and POL), as well as 20 ternary mixture rays and 10 quaternary mixture rays to $V$. qinghaiensis. It was found that the toxicity of IMI does not change with time, those of two ILs both decrease with time, while those of antibiotics both increase with time. The toxicities of all mixture rays, whether ternary or quaternary mixture ray, almost does not change with time, i.e., the mixture toxicity has no time-dependance. However, the toxicological interaction of mixture at different exposure time is different. The ternary mixture rays in [hmim] $\mathrm{Br}-\mathrm{IMI}-\mathrm{CHL}$ and [hmim]Cl-IMI-CHL systems are almost additive action, while most rays in [hmim]Br-IMI-POL and [hmim]Cl-IMI-POL systems exhibit antagonism and the degree of the antagonism has time-dependance. The toxicological interactions of various rays in two quaternary mixture systems verify that $\mathrm{POL}$ is a key component inducing the timedependent antagonism.

\section{Acknowledgements}

The authors are especially grateful to the National Natural Science Foundation of China (21377097).

\section{References}

1 M. C. Newman and J. T. McCloskey, Ecotoxicology, 1996, 5, 187-196.

2 J. H. Lee, P. F. Landrum and C. H. Koh, Environ. Sci. Technol., 2002, 36, 3131-3138.

3 H. A. Tennekes and F. Sanchez Bayo, Toxicology, 2013, 309, 39-51.

4 T. Backhaus and L. H. Grimme, Chemosphere, 1999, 38, 3291-3301.

5 X. W. Zhu, S. S. Liu, H. L. Ge and Y. Liu, Water Res., 2009, 43, 1731-1739.
6 L. Liu, S. S. Liu, M. Yu and F. Chen, Environ. Toxicol. Pharmacol., 2015, 39, 447-456.

7 J. Zhang, S. S. Liu, Z. Y. Yu, H. L. Liu and J. Zhang, J. Hazard. Mater., 2013, 258, 70-76.

8 C. Barata, D. J. Baird, A. J. A. Nogueira, A. Soares and M. C. Riva, Aquat. Toxicol., 2006, 78, 1-14.

9 M. B. A. Bjergager, M. L. Hanson, K. R. Solomon and N. Cedergreen, Aquat. Toxicol., 2012, 110, 17-24.

10 I. Rodea Palomares, F. Leganes, R. Rosal and F. Fernandez Pinas, J. Hazard. Mater., 2012, 201, 209-218.

11 A. S. A. Harabawy and A. T. A. Ibrahim, Ecotoxicol. Environ. Saf., 2014, 103, 61-67.

12 R. Pulicharla, R. K. Das, S. K. Brar, P. Drogui, S. J. Sarma, M. Verma, R. Y. Surampalli and J. R. Valero, Sci. Total Environ., 2015, 532, 669-675.

13 C. M. Aronzon, G. V. Svartz and C. S. P. Coll, J. Chem. Ecol., 2016, 32, 829-842.

14 E. Geiger, R. Hornek Gausterer and M. T. Sacan, Ecotoxicol. Environ. Saf., 2016, 129, 189-198.

15 S. Muthusamy, C. Peng and J. C. Ng, Toxicol. Res., 2016, 5, 703-713.

16 Y. H. Wang, C. Chen, Y. Z. Qian, X. P. Zhao and Q. Wang, J. Hazard. Mater., 2015, 284, 233-240.

17 A. F. Hernandez, T. Parron, A. M. Tsatsakis, M. Requena, R. Alarcon and O. Lopez Guarnido, Toxicology, 2013, 307, 136-145.

18 P. Montuori, S. Aurino, F. Garzonio and M. Triassi, Sci. Total Environ., 2016, 571, 1001-1016.

19 I. Michael, L. Rizzo, C. S. McArdell, C. M. Manaia, C. Merlin, T. Schwartz, C. Dagot and D. Fatta Kassinos, Water Res., 2013, 47, 957-995.

20 K. Kummerer, J. Antimicrob. Chemother., 2003, 52, 5-7.

21 K. Kummerer, Chemosphere, 2009, 75, 417-434.

22 T. P. T. Pham, C. W. Cho and Y. S. Yun, Water Res., 2010, 44, 352-372.

23 M. G. Montalban, J. M. Hidalgo, M. Collado Gonzalez, F. G. D. Banos and G. Villora, Chemosphere, 2016, 155, 405-414.

24 A. A. Camp and D. B. Buchwalter, Aquat. Toxicol., 2016, 178, 49-57.

25 W. H. Xu, G. Zhang, S. C. Zou, X. D. Li and Y. C. Liu, Environ. Pollut., 2007, 145, 672-679.

26 S. Ding, J. W. Wu, M. Zhang, H. F. Lu, Q. Mahmood and P. Zheng, Chemosphere, 2015, 140, 174-183.

27 S. S. Liu, Q. F. Xiao, J. Zhang and M. Yu, Sci. Bull., 2016, 61, 52-58.

28 K. Petersen, H. H. Heiaas and K. E. Tollefsen, Aquat. Toxicol., 2014, 150, 45-54.

29 J. Zhang, S. S. Liu, Z. Y. Yu and J. Zhang, Chemosphere, 2013, 91, 462-467.

30 M. C. Wang, S. S. Liu and F. Chen, Acta Chim. Sin., 2014, 72, 56-60, in Chinese.

31 S. S. Liu, J. Zhang, Y. H. Zhang and L. T. Qin, Acta Chim. Sin., 2012, 70, 1511-1517, in Chinese.

32 L. Feng, S. S. Liu, K. Li, H. X. Tang and H. L. Liu, J. Hazard. Mater., 2017, 327, 11-17. 
33 X. W. Zhu, S. S. Liu, H. L. Ge and Y. Liu, China Environ. Sci., 2009, 29, 113-117, in Chinese.

34 S. S. Liu, X. Q. Song, H. L. Liu, Y. H. Zhang and J. Zhang, Chemosphere, 2009, 75, 381-388.

35 R. Qu, S. S. Liu, F. Chen and K. Li, RSC Adv., 2016, 6, 2101221018.

36 J. Zhang, S. S. Liu, X. Q. Dong and M. Chen, RSC Adv., 2015, 5, 107076-107082.

37 S. S. Liu, K. Li, T. Li and R. Qu, Environ. Int., 2016, 94, 396398.
38 M. J. Jonker, C. Svendsen, J. J. M. Bedaux, M. Bongers and J. E. Kammenga, Environ. Toxicol. Chem., 2005, 24, 2701-2713. 39 M. E. DeLorenzo, G. I. Scott and P. E. Ross, Environ. Toxicol. Chem., 2001, 20, 84-98.

40 R. P. Austin, P. Barton, A. M. Davis, C. N. Manners and M. C. Stansfield, J. Pharm. Sci., 1998, 87, 599-607.

41 J. Ranke, K. Molter, F. Stock, U. Bottin-Weber, J. Poczobutt, J. Hoffmann, B. Ondruschka, J. Filser and B. Jastorff, Ecotoxicol. Environ. Saf., 2004, 58, 396-404.

42 K. A. Heys, R. F. Shore, M. G. Pereira, K. C. Jones and F. L. Martin, RSC Adv., 2016, 6, 47844-47857. 